\title{
Cognitive Procedural Learning in Patients With Fronto-Striatal Lesions
}

\author{
Klaus Schmidtke, ${ }^{1,2,4}$ Hendrik Manner, ${ }^{1}$ Robert Kaufmann, ${ }^{1}$ and Heike Schmolck ${ }^{1,3}$ \\ ${ }^{1}$ Department of Neurology, Freiburg University Clinic, 79106 Freiburg, Germany
}

\begin{abstract}
Previous studies on the role of prefronto-striatal loop systems for cognitive procedural learning (PL) brought inconsistent results. To examine whether the integrity of the dorsolateral prefrontal loop is indispensable for normal cognitive PL, we examined the acquisition of cognitive skills in 35 patients with focal prefrontal lesions, focal caudate lesions, and Huntington's Disease (HD), and compared it with a control group. To examine the potential role of the processing demands made by the cognitive tasks, a set of tasks was applied whose acquisition places demand either on reasoning and problem solving, or on the establishment of fast and repetitive processing routines. The Pursuit Rotor task was also studied with the aim to re-examine earlier findings of a functional segregation of motor and complex prefronto-striatal loops. Deficits of cognitive and motor PL were found to be limited to certain tasks and groups. PL of one task, which demanded rapid, repetitive processing of visuo-spatial stimuli, was impaired in all patient groups. PL of two problem-solving tasks was impaired in patients with focal and degenerative caudate lesions only. None of the groups was impaired at PL of Mirror Reading, another task demanding rapid and repetitive visuo-spatial processing, and none was impaired in a fifth task of language skill. Deficits of motor learning were only observed in patients in whom the motor loop was affected. These results suggest that the dorsolateral prefronto-striatal loop is involved in the establishment of cognitive processing routines. The comparison of patients with and without caudate lesions suggests that disconnection of this circuit is critical. However, the normal acquisition of two cognitive tasks even in this group indicates that the integrity of the dorsolateral prefrontal loop is not mandatory for normal cognitive PL. We discuss these dissociations with regard to the demand profiles of the applied tasks and the locations of the lesions.
\end{abstract}

Procedural learning (PL), or skill learning, means acquisition and improvement of skills through practice. PL is a heterogeneous phenomenon that includes cognitive, perceptual, motor, and other skills. Its results can only be demonstrated by improvement of performance. PL is, however, not implicit; subjects are usually aware that they are about to acquire a new skill. A subset of PL tasks is purely nondeclarative, whereas the acquisition of other skills is superimposed by declarative learning of certain steps and procedures (e.g., chess or driving). A pivotal feature of cognitive PL is that transfer takes place, that is, new cognitive skills are applicable to new problems or new versions of problems of the class that were processed during previous learning.

Knowledge of the neuronal basis of PL is limited. Neuropsychological studies have shown dissociations in the learning of skills in patients with different brain pathologies (Brooks and Baddely 1976; Beatty et al. 1987; Harrington et al. 1990). Findings in patients with basal ganglia disease led

Present addresses: ${ }^{2}$ Center for Geriatric Medicine and Gerontology, Freiburg University Clinic, 79106 Freiburg, Lehener Str. 88, Germany; ${ }^{3}$ Department of Neurology, Baylor College of Medicine, Houston, TX 77030, USA.

${ }^{4}$ Corresponding author.

E-MAIL schmidtk@zggf.ukl.uni-freiburg.de; FAX 0049-7612707089.

Article and publication are at http://www.learnmem.org/cgi/doi/ 10.1101/lm.47202. to the theory of a neostriatal habit learning system, composed of the neostriatum and associated prefrontal structures (DeLong et al. 1983; Butters et al 1985; Heindel et al. 1989). One formulation for the role of the basal ganglia and associated prefrontal structures is that they subserve the stepwise generation of new, successful modes of behavior, specifically in the early stage of cognitive learning (Saint-Cyr and Taylor 1992; Saint-Cyr et al. 1992, 1995).

Anatomically, the striatum is closely connected to the prefrontal cortex (DeLong et al. 1983, Alexander et al. 1986, 1990; Alexander and Crutcher,1990). Their association is based on partially closed cortico-striato-pallido-nigrothalamo-cortical loop systems. There is a motor loop, an oculomotor loop, and three complex loops. The caudate nucleus serves as a relay for two of the complex loops, namely the dorsolateral prefrontal loop, which includes the prefrontal Brodmann Areas (BA) 9 and 10, parts of the premotor cortex and parietal BA 7, and the lateral orbito-frontal loop, which includes the inferior parts of prefrontal BA 10 and 11 and parts of the temporal neocortex. The anterior cingulate loop includes BA 24, temporal limbic areas and the ventral striatum (for review, see Alexander and Crutcher 1990; Saint-Cyr and Taylor 1992; Saint-Cyr et al. 1992; Cummings 1993). The caudate nucleus degenerates in Huntington's Disease (HD), and it suffers dopaminergic differentiation in Parkinson's Disease (PD), the degree of

LEARNING \& MEMORY 9:419-429 @ 2002 by Cold Spring Harbor Laboratory Press ISSN1072-0502/02 \$5.00

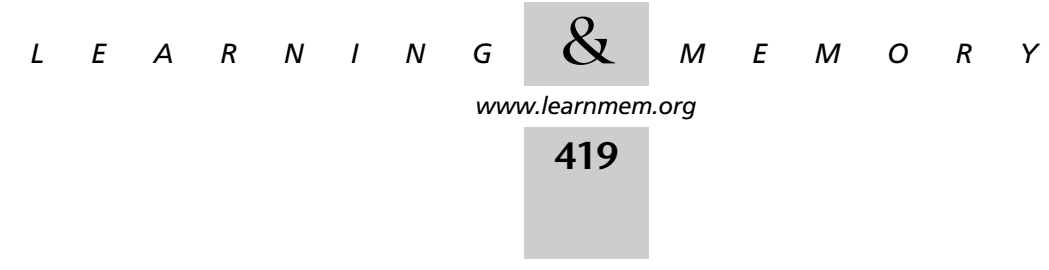


which is correlated with measures of cognitive impairment (Holthoff-Detto et al. 1997). The putamen belongs to the motor loop and is affected by both HD and PD.

Positron emission tomography studies showed an increase of metabolic activity in the basal ganglia and the prefrontal association cortex during the execution of noncognitive procedural tasks (Perani et al. 1993), and activation of the left caudate nucleus during learning of an implicit sequence (Peigneux et al. 2000). Dagher et al. (1999) examined cerebral activation during the execution of the problem-solving task Tower of London, after subjects had received 30-min training. They found evidence for involvement of the dorsolateral prefrontal cortex and the right caudate nucleus, among other structures, in the execution of the task. In regard to motor PL, several functional magnetic-resonance imaging (fMRI) studies have shown a temporary activation of prefrontal and premotor areas during the acquisition of new skills (van Mier et al. 1998). Another fMRI study showed activation of the right caudate nucleus during execution of a cognitive task (Poldrack et al. 1999). However, it has not been demonstrated that these activations subside with increasing skill. Because trained performance is usually less effortful, one would expect a decreased rather than increased functional activation. Another possibility is that one neuronal system assists another during the early stage of PL and is thus only temporarily active. Furthermore, processing may shift from one system to another during PL, for example, from a controlled, workingmemory-dependent mode to a more automatic and taskspecific mode that relies on associative cortex. In summary, functional imaging studies have shown an involvement of fronto-striatal structures in learning, but it is not yet clear to what degree these activations represent learning in the strict sense, that is, dynamic changes of processing, or rather task-specific processing that is constant and independent of the stage of learning.

Some clinical findings cast doubt on the universal importance of the prefronto-striatal systems for PL. In patients with prefrontal lesions, PL of the serial reaction-time task was impaired (Beldarrain et al. 1999), whereas PL of mirror reading was normal in another study (Daum et al. 1995). Normal PL of mirror reading was also found in Korsakoff patients, in whom neuronal damage typically involves the ventral anterior and dorsomedial thalamic nuclei, which are relay structures of the three complex loops (Schmidtke et al. 1996). Findings in degenerative diseases are also equivocal. In PD, some cognitive tasks were found to be learned in a normal fashion, for example, an artificial grammar task (Reber and Squire 1999), whereas others were not, for example, a variant of the Tower of Hanoi task (Saint-Cyr et al. 1988, Daum et al. 1995). PL of the mirror reading skill was reported to be impaired by Roncacci et al. (1999) and Koenig et al. (1999), but intact by Harrington et al. (1990). In HD, Martone et al. (1984) found a mild PL deficit in a mirror reading task. Gabrieli et al. (1987) reported intact PL of a mirror-tracing skill. Another point is that PD and HD are imperfect models of striatal damage, because they cause neuronal damage beyond the basal ganglia (De la Monte et al. 1988, Sotrel et al. 1991).

The present neuropsychological group study was exploratory in nature. Its main goal was to investigate whether the integrity of the dorsolateral prefrontal loop system is mandatory for cognitive PL. Because HD and PD can lead to neuronal dysfunction outside of the basal ganglia, we studied groups of patients with focal unilateral prefronto-striatal lesions, either to the head of the caudate nucleus or to the dorsolateral prefrontal association cortex. In addition, a group of HD was included. Thus, patients with three different types of fronto-striatal pathology were examined.

The second goal was to investigate effects of task type on the efficiency of cognitive PL in patients with frontostriatal lesions. Previous studies had reported variable results by use of different cognitive tasks. We assumed that a reason for this variability could be the different demands made by different tasks and the impact of fronto-striatal dysfunction on the cognitive processes that are involved. Some cognitive tasks require reasoning and development of new strategies during the early stage of learning, whereas others involve little reasoning, but the establishment of fast and repetitive processing routines, that is, specialized working memory capacities. Both reasoning and working memory are attributed to the group of executive functions that are related to the prefrontal cortex. In other words, deficits of cognitive PL could potentially be attributed to deficits in reasoning and problem solving, and/or to deficits in the establishment, automatization, and acceleration of specialized working memory capacities, or to neither of those. We intended to investigate these possibilities by applying tasks that require reasoning, problem solving, and strategic thinking, and tasks that require rapid, repetitive processing of visuo-spatial stimuli, but little strategy. Two tasks each were selected, one predominantly verbal and one predominantly nonverbal. As a reference, a fifth task was included that requires neither reasoning and strategy, nor fast and repetitive processing.

The third part of the study was essentially confirmatory; we intended to re-examine earlier findings regarding the functional segregation of fronto-striatal loop systems. To this end, a visuo-motor skill was included. PL of this task was compared in patients with lesions limited to the complex loops versus patients with additional lesions to the motor loop.

\section{RESULTS}

\section{Procedural Learning}

Mean absolute PL task performances at baseline and at the final trial are listed in Table 1 . In addition, mean perfor-

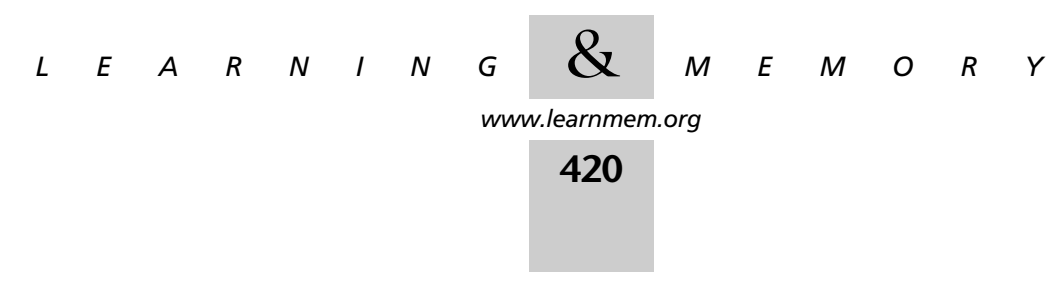


Table 1. Mean Absolute Performances at the Procedural Learning Tasks at the $1^{\text {st }}$ and the $3^{\text {rd }}$ Trial

\begin{tabular}{|c|c|c|c|c|}
\hline & $\begin{array}{l}\text { Control } \\
\text { Group } 1\end{array}$ & $\begin{array}{l}\text { Prefrontal } \\
\text { lesions }\end{array}$ & $\begin{array}{l}\text { Caudate } \\
\text { lesions }\end{array}$ & $\begin{array}{l}\text { Huntington's } \\
\text { disease }\end{array}$ \\
\hline Tower of Hanoi, $1^{\text {st }}$ trial $/ 2^{\text {nd }}$ trial (moves $>15$ ) & $7.4 / 4.4$ & $12.7 / 4.6$ & $13.7 / 10.2$ & $7.9 / 7.0$ \\
\hline Logical Reasoning, $1^{\text {st }}$ trial $/ 3^{\text {rd }}$ trial (seconds) & $371 / 250$ & $572 / 394$ & $348 / 280$ & $540 / 419$ \\
\hline Sentence Arrangement, $1^{\text {st }}$ trial $/ 3^{\text {rd }}$ trial (seconds) & $347 / 209$ & $431 / 302$ & $702 / 363$ & $594 / 417$ \\
\hline Mental Rotation, $1^{\text {st }}$ trial $/ 3^{\text {rd }}$ trial (seconds) & $236 / 153$ & $352 / 291$ & $265 / 227$ & $413 / 348$ \\
\hline \multirow[t]{2}{*}{ Mirror Reading, $1^{\text {st }}$ trial $/ 3^{\text {rd }}$ trial (seconds) } & $426 / 227$ & $683 / 318$ & $476 / 294$ & $948 / 441$ \\
\hline & $\begin{array}{l}\text { Control } \\
\text { Group } 2\end{array}$ & $\begin{array}{l}\text { Prefrontal } \\
\text { lesions }\end{array}$ & $\begin{array}{l}\text { Caudate } \\
\text { lesions }\end{array}$ & $\begin{array}{l}\text { Huntington's } \\
\text { disease }\end{array}$ \\
\hline $\begin{array}{l}\text { Pursuit Rotor, adjusted number of rounds per minute } \\
\text { Pursuit Rotor, time off-target (means of } 1^{\text {st }} \text { to } 3^{\text {rd }} / 4^{\text {th }} \text { to } 6^{\text {th }} \text { trial) }\end{array}$ & $\begin{array}{c}16.5 \\
19.9 / 13.6\end{array}$ & $\begin{array}{c}15.5 \\
21.6 / 15.9\end{array}$ & $\begin{array}{l}17.7 \\
23.7 / 18.6\end{array}$ & $\begin{array}{c}15.0 \\
26.3 / 21.9\end{array}$ \\
\hline
\end{tabular}

Lower values indicate better performance. For Tower of Hanoi, time to completion, see Fig. 2.

mances in the Tower of Hanoi task (time to completion) are shown in Figure 2, below.

Table 2 lists the mean PL scores for the three patient groups and the control group in the procedural learning tasks, as well as the significance level of group differences between patients and controls, where present. Differences between controls' and patients' mean PL scores were evaluated by a two-sided t-Test for the patient groups with prefrontal and caudate lesions. Due to the difference in mean age, analysis of covariance with age as a covariate was applied in the case of HD patients. Examples of the distribution of final and baseline scores in two PL tasks are given in Figure 1.

In the prefrontal group, laterality effects on PL were very small and nonsignificant in all of the PL tasks. In the caudate group, laterality effects were not examined due to the limited case number (an exception is Pursuit Rotor, see below).

\section{Effects of Cognitive Abilities and Age on Procedural Learning}

To examine the effects of visuo-spatial ability, frontal/executive capacity and age on cognitive PL, Pearson's correlation coefficients between the corresponding variables and PL scores were calculated. This was done taskwise within the group of controls and within the group of all frontostriatal patients. If significant correlations were found, the corresponding variables were entered as covariates in analyses of variance of PL scores. This was carried out to examine whether group differences of PL, where present, were related to group differences of visuo-spatial ability or word fluency.

Visuo-spatial ability was significantly $(P<0.005)$ correlated with controls' PL scores in Logical Reasoning $(r=-0.39)$, Tower of Hanoi, time $(r=-0.40)$, Mental Rotation $(r=-0.50)$ and Mirror Reading $(r=-0.48)$, and with

Table 2. Mean Procedural Learning Scores (Quotients of Post-training and Baseline Performances)

\begin{tabular}{|c|c|c|c|c|}
\hline & $\begin{array}{l}\text { Control } \\
\text { Group } 1\end{array}$ & $\begin{array}{l}\text { Prefrontal } \\
\text { lesions }\end{array}$ & $\begin{array}{c}\text { Caudate } \\
\text { lesions }\end{array}$ & $\begin{array}{c}\text { Huntington's } \\
\text { disease }\end{array}$ \\
\hline Tower of Hanoi, time ( $1^{\text {st }}$ to $2^{\text {nd }}$ trial) & 0.37 & 0.37 & $0.57\left(^{*}\right)$ & $0.64^{* *}$ \\
\hline Tower of Hanoi, moves ( $1^{\text {st }}$ to $2^{\text {nd }}$ trial $)$ & 0.51 & 0.50 & $0.63^{*}$ & $0.59^{*}$ \\
\hline Logical Reasoning & 0.70 & 0.75 & $0.86\left(^{*}\right)$ & $0.80\left(^{*}\right)$ \\
\hline Sentence Arrangement & 0.74 & 0.79 & 0.73 & 0.84 \\
\hline Mental Rotation & 0.55 & $0.69 *$ & $0.77^{* *}$ & $0.78^{* * *}$ \\
\hline \multirow[t]{2}{*}{ Mirror Reading } & 0.45 & 0.42 & 0.51 & 0.43 \\
\hline & $\begin{array}{l}\text { Control } \\
\text { Group } 2\end{array}$ & $\begin{array}{l}\text { Prefrontal } \\
\text { lesions }\end{array}$ & $\begin{array}{c}\text { Caudate } \\
\text { lesions }\end{array}$ & $\begin{array}{c}\text { Huntington's } \\
\text { disease }\end{array}$ \\
\hline Pursuit Rotor & 0.75 & 0.80 & $\begin{array}{c}0.85 \\
\text { (see Discussion for subanalysis) }\end{array}$ & $0.88^{*}$ \\
\hline
\end{tabular}

Lower values indicate better learning. Differences of controls' and patients' means were evaluated by two-sided t-test in patients with prefrontal and caudate lesions and by analysis of covariance, with age as a covariable, in HD patients (see Materials and Methods). $\left.{ }^{*}\right)$ Approaching statistical significance $(P<0.07),{ }^{*} p<0.05,{ }^{* *} p<0.005, * * * p 0.0005$.

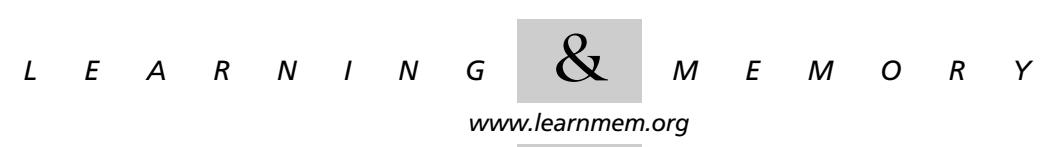



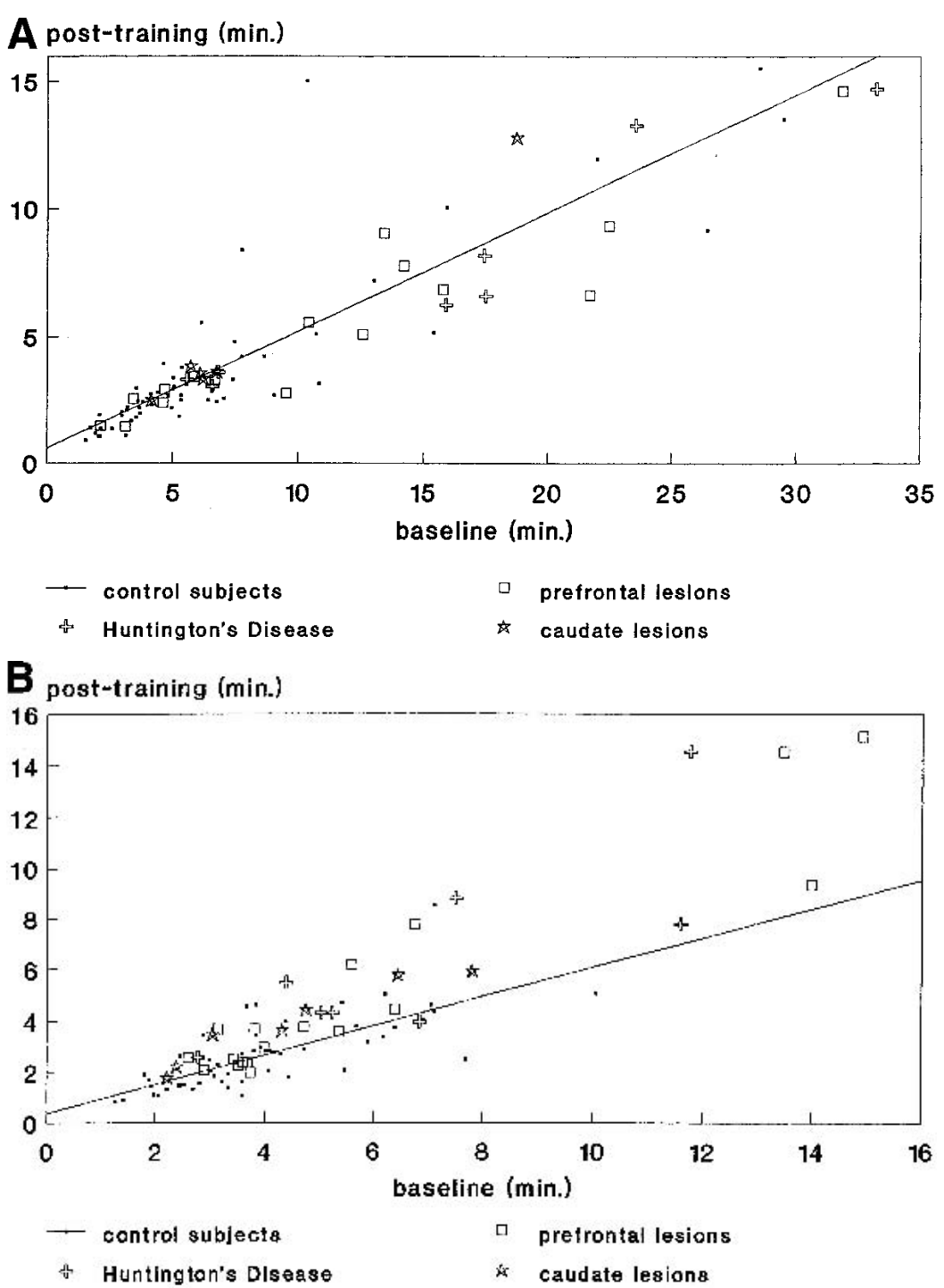

Figure 1 Scatterplots of baseline performances vs. post-training performances of two cognitive task examples. Figures illustrate the difference of procedural learning between two tasks. Continuous lines represent the regression lines of the control subjects' data. Data points above the regression line indicate lower-than-average PL. Note the close linear relationship between baseline and third trial performances, despite large interindividual differences of baseline performance and of improvements after training. $(A)$ Mirror Reading. The two patients with the lowest baseline performance (highest number of minutes) were not included in the analysis of PL (see also Materials and Methods). (B) Mental Rotation. The five patients with the lowest baseline performance were not included in the analysis of PL.

patients' PL scores in Mirror Reading only $(r=-0.36$, $P=0.05)$. However, regression analyses of controls' $\mathrm{PL}$ scores, with visuo-spatial ability and age as independent variables, identified age as the only significant determinant of learning in these tasks. When visuo-spatial ability was entered as a covariate in analyses of covariance of PL scores (each patient group vs. the control group), between-group differences of PL, where present, remained significant.

Word fluency was significantly correlated with con- trols' but not with patients' PL scores in two tasks, that is, Mirror Reading $(r=-0.29, P=0.042)$ and Mental Rotation $(r=-0.38, P=0.006)$. In Mirror Reading, a regression analysis of PL scores with word fluency and age identified only age as a significant determinant. In Mental Rotation, an analogous regression analysis identified both variables as significant determinants. Both variables were then entered as covariates in analyses of covariance of PL scores (each patient group vs. the control group). The present differences between patient group and control group in Mental Rotation remained significant.

Age was significantly correlated with controls' PL scores in Logical Reasoning $(r=-0.43)$, Tower of Hanoi, time $(r=-0.45)$, Mental Rotation $(r=-0.56)$ and Mirror Reading $(r=-0.61)$. For the results of regression analyses with age, visuospatial ability, and word fluency, see above.

\section{Effects of Cognitive Abilities and Age on Absolute Performance in Procedural Learning Tasks}

Changes in the relationship between task performance and cognitive abilities can provide information about changing modes of processing during PL. Therefore, correlations between cognitive abilities, age, and absolute PL task performance were calculated in the group of controls and in the group of all patients.

In the control group, a high and significant $(P<0.0005)$ correlation was present between visuo-spatial ability and absolute performances in three cognitive tasks, that is, Logical Reasoning, Mental Rotation, and Mirror Reading, both at baseline $(r=-0.51$ to -0.66$)$ and in the final trial $(r=-0.64$ to $-0.67)$. In the patient group, an analogous pattern was found at baseline $(r=-0.56$ to $-0.62)$, and in the final trial $(r=-0.57$ to $-0.64)$. Regression analyses with visuo-spatial ability, word fluency, and age as independent variables confirmed visuo-spatial ability as a significant determinant. Age was an additional determinant for controls' final performance in two tasks (Logical Reasoning and Mirror Reading).

Lower, but significant correlations were present between visuo-spatial ability and Tower of Hanoi, time at baseline ( $r=-0.32$ in controls, $r=-0.41$ in patients). Regression analyses confirmed this variable as the only determi-

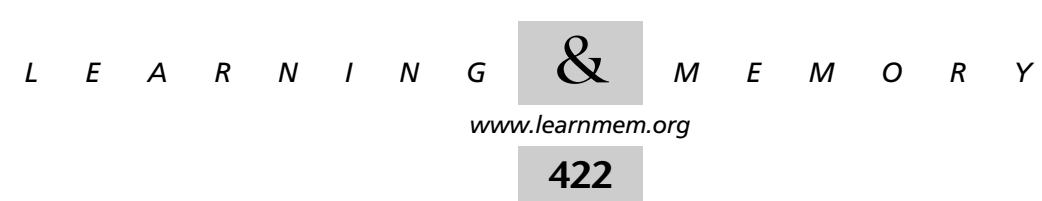


nant. During trials 2 to 6 , the correlations with word fluency increased in both groups, and regression analyses of sixth trial performances identified word fluency (patient group) and word fluency and age (control group) as the only determinants. Correlations with the baseline number of moves were not significant.

A similar pattern was observed in Pursuit Rotor, in which the only determinant of patients' mean performance during the first block of trials was visuo-spatial ability, and word fluency during the second block. The correlation with visuo-spatial ability dropped from $r=-0.53$ to -0.35 ; the correlation with word fluency remained nearly stable ( $r=-0.45$ to -0.49 ). The speed of the rotating stimulus, which was adjusted individually to 10,15 , or 20 rounds per minute before the experiments (see below), was correlated with visuo-spatial ability in patients $(r=0.49, P=0.003)$ and control subjects $(r=0.51, P<0.001$; rank correlations). Rank correlations with the other variables were lower and/or not significant.

\section{DISCUSSION}

This study examined the acquisition of five cognitive skills and a visuo-motor skill in subjects with focal prefrontal lesions, focal caudate lesions, and HD. Performances below control level that patients exhibited on standard neuropsychological tests (Table 3) and at the baseline trials of the PL tasks (Table 1) are likely to be related to a frontal-dysexecutive syndrome that results from the present fronto-striatal lesions. Two patients with extensive left-frontal gliomas that involved the anterior corpus callosum could not solve the Tower of Hanoi puzzle and had to be excluded from the analysis of PL in this task.

\section{Cognitive Tasks}

The control groups made considerable progress during the training of the PL tasks. Mean performances improved by $-30 \%$ to $60 \%$ (Tables 1 and 2). In comparison with the control group, patients with prefrontal and striatal lesions showed significant deficits of cognitive and motor PL. However, these deficits were limited to certain tasks and groups (see Table 2). Two example raw data scatterplots illustrate the variability of learning between and within the subject groups (Fig. 1).

In two tasks, Mirror Reading and Sentence Arrangement, all three patient groups exhibited normal PL (Table 2). In Mirror Reading, the mean PL learning scores of prefrontal patients and HD were nearly identical to those of control subjects; the score of patients with focal caudate lesions was slightly and nonsignificantly worse (see also Fig. 1A). In Sentence Arrangement, a minor and nonsignificant deficit was seen in HD patients. Normal PL of Mirror Reading tasks was found earlier in a study with PD patients (Harrington et al. 1990). Others reported deficits in PD patients (see above). One study that used a different method for the assessment of PL found a mild deficit in HD, although learning within the first block of trials (day 1) and progress from day 1 to day 2 was normal (Martone et al. 1984). Our results with these two tasks demonstrate that the integrity of the complex prefronto-striatal systems, including the caudate nuclei, is not mandatory for normal PL of cognitive skills. This conclusion applies to the three types of fronto-striatal lesion that were examined, that is, unilateral vascular and bilateral degenerative caudate lesions, and lesions to the dorsolateral prefrontal association cortex. It is possible that larger or bilateral prefrontal and caudate lesions, or more advanced HD, would have caused an impairment. However, the present patient groups were quite impaired at baseline (Table 1), which indicates that the extent of their lesions was sufficient to cause a disruption of cognitive functions. Furthermore, there was a dissociation in that the three patient groups exhibited marked and statistically significant PL deficits in other tasks.

In Mental Rotation, a pronounced deficit was present in all patient groups, suggesting that the dorsolateral prefrontal loop is part of a neuronal circuitry that is required for this type of learning. Prefrontal patients were relatively less impaired (Table 2; Fig. 1B). In the two tasks of reasoning and problem solving, Logical Reasoning and Tower of Hanoi (time and moves, first to second trial), the HD and caudate groups showed variable PL deficits, which approached or reached statistical significance (Table 2). In the third and fourth trial of the Tower of Hanoi task, all groups,

Table 3. Demographical Data and Mean Performances at Standard Neuropsychological Tests

\begin{tabular}{lccccc}
\hline & $\begin{array}{c}\text { Control Group 1, } \\
n=56\end{array}$ & $\begin{array}{c}\text { Control Group 2, } \\
n=35\end{array}$ & $\begin{array}{c}\text { Prefrontal } \\
\text { lesions, } \\
n=20\end{array}$ & $\begin{array}{c}\text { Caudate } \\
\text { lesions, } \\
n=7\end{array}$ & $\begin{array}{c}\text { Huntington's } \\
\text { disease, } \\
n=8\end{array}$ \\
\hline Mean age and age range & $46.9(18-78)$ & $56.6(23-76)$ & $51.9(21-70)$ & $50.4(35-61)$ & $40.0(25-52)$ \\
Sex & $32 \mathrm{~m}, 24 \mathrm{f}$ & $18 \mathrm{~m}, 18 \mathrm{f}$ & $8 \mathrm{~m}, 12 \mathrm{f}$ & $7 \mathrm{~m}$ & $6 \mathrm{~m}, 2 \mathrm{f}$ \\
Word fluency & 109.4 & - & 78.5 & 67.6 & 71.1 \\
Visuo-spatial ability & 21.1 & - & 15.7 & 15.8 & 16.1 \\
Mean scaled score of three WAIS-R subtests & 11.3 & - & 59.5 & 58.1 & 7.6 \\
Naming (maximum score: 60) & - & & & &
\end{tabular}

See Materials and Methods section for description of tests.

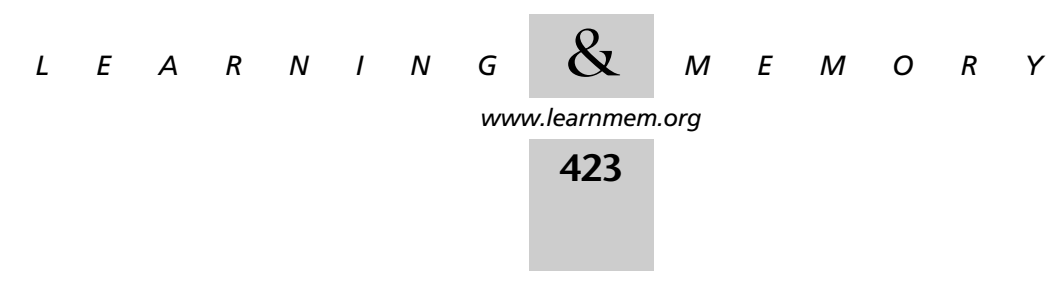


including prefrontal patients, but not controls, suffered a relapse, which shows that the new skill was not yet consolidated to a normal extent (Fig. 2). In the fifth and sixth trials, all groups made further progress, and the prefrontal group approached the level of the controls.

The present differences of results across subject groups and tasks suggests a relevance of the type of processing for successful acquisition of cognitive skills. Previously, we referred to the potential relevance of reasoning and problem solving versus establishment of fast and repetitive processing routines. Three findings indicate that this distinction does not constitute the divide between normal and impaired PL. First, PL deficits of the problem-solving tasks were not uniform across the three patient groups. Second, PL of both types of tasks was impaired in the caudate and HD group. Third, all patient groups were impaired in the learning of Mental Rotation, but unimpaired in the learning of Mirror Reading, two tasks that we attributed to the same type. The latter finding is remarkable given the apparent similarity of the two tasks. Both involve fast, multiple-item, visually guided processing that requires visual working memory and mental transformation of symbols. Both show a similar, significant correlation between absolute performance and visuo-spatial ability scores.

An alternative explanation for the present dissociation is that learning of Mirror Reading depends predominantly on specialized object recognition and object transformation networks that are localized in posterior, temporo-parietooccipital areas, whereas PL of Mental Rotation relies on improvement of a specialized working memory capacity, that is, rapid comparison of two visual stimuli, which is functionally related to prefronto-neostriatal circuits. This idea is supported by the finding that word fluency, thought
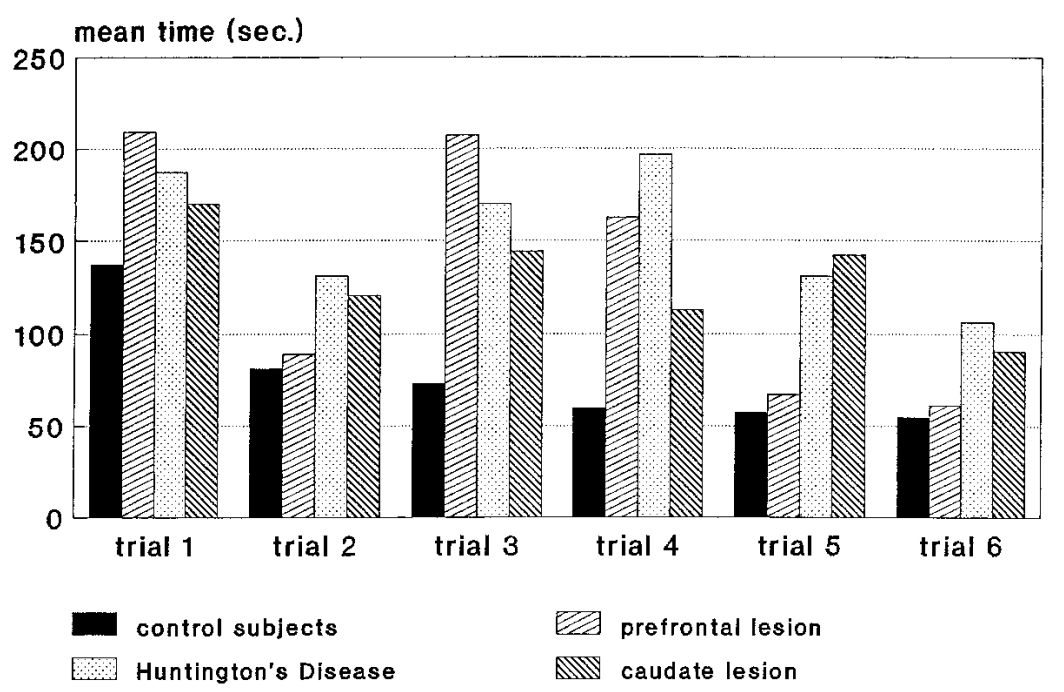

Figure 2 Mean performances in the Tower of Hanoi task (time to completion). Each of the six sets of columns represents the mean performance of the four subject groups at one trial. Two trials were performed per day. to be related to prefrontal function, was an independent determinant of controls' PL scores in Mental Rotation, but not in Mirror Reading. Furthermore, evidence from earlier fMRI studies suggests that learning of Mirror Reading does occur through functional adaptation of posterior association areas (Poldrack et al 1998; Kassubek et al. 2001). This line of interpretation could explain why the Sentence Arrangement task was learned in a normal fashion; temporal and premotor areas of the left hemisphere, which are involved in language processing, remained essentially intact in all patient groups.

In addition to the question of task type, it is obvious that the location of fronto-striatal lesions played a role. Patients with caudate damage, that is, focal vascular lesion and bilateral degenerative lesions, were significantly impaired in the reasoning and problem solving tasks, whereas the group with prefrontal lesions was not, except for the relapse seen in the third and fourth trials of the Tower of Hanoi task. Prefrontal patients were also less impaired in the learning of Mental Rotation (Table 2). This suggests that the integrity of the caudate nucleus is of particular importance. If one assumes that the dorsolateral prefrontal loop serves as adaptive neuronal circuitry for PL of certain cognitive tasks, then the caudate nucleus is an anatomical bottleneck of this circuitry.

In summary, some, but not all patients with frontostriatal lesions exhibited PL deficits in reasoning and problem-solving tasks, and all were impaired in Mental Rotation, a task that requires improvement of a specialized working memory capacity. This suggests that dysfunction of the dorsolateral prefrontal loop may lead to PL deficits by means of impairment of reasoning and problem solving, or by means of a deficit in the automatization and acceleration of specific working memory processes. It is of interest that bilateral activation of the dorsolateral prefrontal cortex was shown in subjects who worked on a problem-solving task comparable with the Tower of Hanoi (Baker et al. 1996), whereas our patients, whose prefrontal cortex had suffered considerable lesions, showed only minor and nonsignificant impairments in the problem-solving tasks. We found lesions to the caudate nuclei, which disconnect the prefrontal cortex, to be more critical despite incomparably lower lesion volume.

It should be pointed out that, with two exceptions (see above), all patients were able to perform the tasks, and that learning was analyzed in relation to their individual baseline performances. Nevertheless, the results reported here may be influenced by the way PL was assessed. The principal question is which method is best to com-

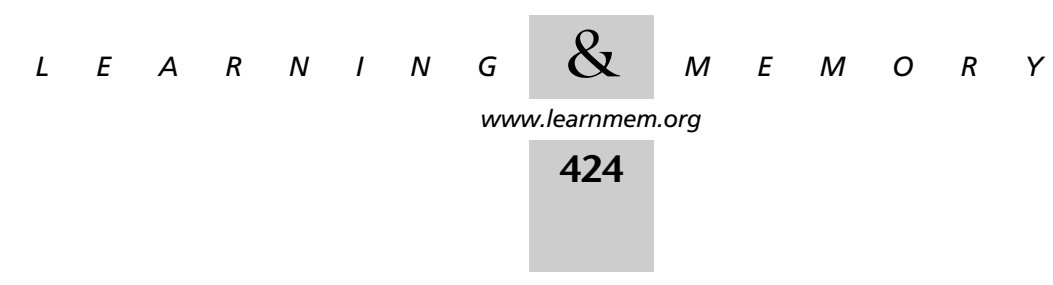


pare learning in subjects with different baseline performances? The approach applied here is based on quotients of performances. An alternative would have been to train low-performing subjects to control level and compare learning from there on. We did so in the Pursuit Rotor task, but consider this approach to be less suitable in cognitive learning. This type of learning occurs in a succession of qualitatively different steps (Anderson 1982). If one gives patients the opportunity to improve to control level, a substantial bias could result, because they might go through early stages of learning before the start of the main experiment. Another possible approach is a difference score. However, it is evident that the meaning of numerically identical improvements is quite different at opposite ends of the baseline performance range. Another methodological point is that clinical case groups are never completely homogeneous. The present findings may have been influenced by involvement of the anterior limb of the internal capsule in some patients with caudate damage, by cortical involvement in HD, by different sizes of the prefrontal lesions, and by involvement of the medial frontal cortex in some patients. In regard to the prefrontal group, mean learning scores were-except for one task-either minimally lower than control level, or exactly at control level. This suggests that lesions transgressing the dorsolateral cortex played no important role (Table 2).

\section{Pursuit Rotor Task}

PL was severely impaired in the HD group, as observed earlier in comparable versions of the task (Gabrieli et al. 1987; Heindel et al. 1989). Involvement of the striatum during rotary pursuit has also been suggested by PET findings in normal subjects (Grafton et al. 1992). In caudate lesions, the motor loop is essentially intact, and Pursuit Rotor learning should proceed normally. However, in the four of our seven patients with left-side caudate lesions (all of whom performed the task with the right hand), the putamen was additionally involved, and a post-hoc analysis showed that these four patients attained a highly pathological mean learning score of 0.90 (control group, 0.75; HD group, 0.88). In contrast, the remaining three subjects whose lesions were on the right side and limited to the head of the caudate nucleus attained a near-normal mean PL score of 0.79. In the group of patients with prefrontal lesions, PL of Pursuit Rotor was unimpaired. Their lesions involved the dorsolateral prefronto-striatal loop system and, in a subgroup of cases, the anterior cingulate loop. The present profile is in line with a functional segregation of frontostriatal loop systems with regard to cognitive versus motor PL. Lesions of the dorsolateral prefrontal cortex leave the motor loop intact, whereas in HD, striatal degeneration affects the caudate and putaminal nucleus bilaterally, and thus impairs complex and motor loop systems.

\section{The Role of Cognitive Abilities, Age, and Laterality}

Patients were impaired in regard to word fluency and visuospatial ability. Only minor and nonsignificant differences were present between the three groups (Table 3). In many cases, patients' baseline performances in the PL tasks were also impaired (Table 1). A comparison between patient groups reveals no group- or domain-specific pattern in the distribution and extent of these impairments.

Visuo-spatial ability was significantly correlated with controls' PL scores in the cognitive tasks, excluding the one purely verbal task. However, regression analyses did not identify visuo-spatial ability as an independent determinant of PL. It is therefore not surprising that coexisting deficits of visuo-spatial ability cannot explain patients' deficits of cognitive PL, as demonstrated by analyses of covariance. Visuospatial ability was, however, identified as a highly correlated, significant determinant of absolute performance in the same PL tasks. This is explained by the fact that execution of these tasks involves the manipulation of visual material and representational mental images. An example is Mental Rotation, considered to be a prototypical visuo-spatial task, in which visuo-spatial ability was the only significant determinant of baseline and final performances in control and patient groups, but not a determinant of PL scores. Visuo-spatial ability was clearly impaired in all patient groups, but this impairment was not related to patients' PL deficits in this task. The finding that PL was unrelated to visuo-spatial ability in all tasks despite high correlations with absolute performances emphasizes that cognitive and neuronal processes involved in the acquisition and in the execution of cognitive tasks can be different. In other words, learning and doing can depend on different neuronal mechanisms and/or cognitive processes.

Word fluency, applied as a measure of frontal/executive function, was reduced by $-30 \%-40 \%$ in patients compared with the control group. It was identified as an independent determinant of controls' PL scores in one task, Mental Rotation. However, patients' word fluency deficits did not explain their deficits in the learning of this task. This suggests that no close functional relationship exists between the mechanisms that are involved in word generation and those involved in the acquisition of this task.

In the Tower of Hanoi task (time to completion), the pattern of correlations with absolute performance changed from the first to the sixth trial in both patients and controls. Visuo-spatial ability was the only determinant of performance in the first trial, whereas word fluency and age were determinants in the sixth trial. This pattern suggests that spatial analysis is involved in naive performance of task, whereas executive functions like speed and accuracy of processing play a dominant role for skilled performance. A similar transition was observed in the visuo-motor Pursuit Rotor task, in which visuo-spatial ability was the only deter-

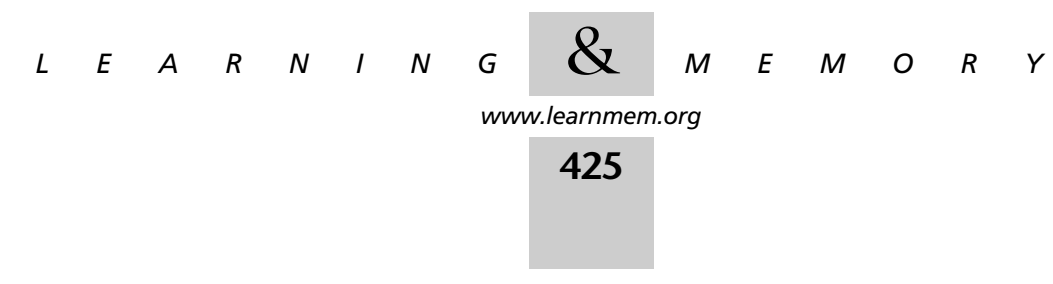


minant of patients' performance in the first block, and word fluency in the second block.

Age was not a determinant of absolute baseline performance in any group or task. In the control group, however, it was an independent determinant of PL in four of five cognitive tasks (Logical Reasoning, Tower of Hanoi / time, Mental Rotation, and Mirror Reading). Older subjects generally learned at a slower pace. This suggests that the acquisition of PL tasks is more age dependent than their execution, that is, that the adaptability of the nervous system is, in this respect, disproportionately more sensitive to the effects of aging. The finding that age was also a determinant of controls' absolute post-training performance in some tasks is a sequel of its inverse effect on learning (Logical Reasoning, Tower of Hanoi/time, Mirror Reading). The fact that the patients were significantly younger than control group 2 is not relevant given that age was not a determinant of learning in the Pursuit Rotor task.

Effects of declarative memory impairment on PL were not examined, because a previous study in a group of 20 amnesic patients showed no correlation between residual declarative memory capacity and PL scores in any of the cognitive tasks that were applied here. There was indirect evidence for a role of declarative memory in one task, Tower of Hanoi, in that absolute performances were highly correlated with memory scores in these patients. Hence, it remains possible that declarative memory deficits contributed to PL deficits of patients with HD and caudate lesions in this task. The laterality of the lesions played no role for cognitive PL in any task (only prefrontal patients examined).

\section{MATERIALS AND METHODS}

\section{Fronto-Striatal Patients}

Three groups of patients with fronto-striatal lesions were studied, unilateral lesions to the dorsolateral prefrontal association cortex, unilateral focal lesion to the head of the caudate nucleus, and Huntington's Disease. All patients were free of previous or additional CNS disease and of current substance abuse. Only minor and nonsignificant differences in mean age were present between control group 1 and patients with prefrontal and focal caudate lesions (Table 3). In HD patients, mean age was $7 \mathrm{yr}$ lower than in control group $1(P=0.083)$. Mean visuo-spatial ability and word fluency scores were clearly impaired in comparison with control group 1 , whereas differences between patient groups were minor and nonsignificant. Patients' mean scaled scores of three WAIS-R-subtests were below the value of the control group 1 , but within the range of one standard deviation of the mean, that is, $10+/-3$ (Table 3 ). Naming was generally unimpaired. Neuropsychological measures are explained at the end of this section.

A difference in mean age between the group of all patients (48.9 yr) and control group 2 (56.6 yr) was significant.

Twenty patients, eight men and twelve women, had a unilateral lesion to the dorsolateral prefrontal cortex (nine right, eleven left). Age ranged from 21 to $70 \mathrm{yr}$. The delay between onset of symptoms or surgery and inclusion in the study ranged from days to years. The lesions were due to the following etiologies, tumor in 14 cases, either pre- or postoperative (glioma $n=8$, meningioma $n=5$, metastasis of bronchial cancer $n=1$ ), ischemic stroke in four cases, hemorrhage and trauma in one case each. The size and location of the lesions, as visualized by computed tomography and/or magnetic-resonance imaging, are listed in Table 4. The lesions affected the cortex and the white matter of the dorsolateral prefrontal cortex [Brodmann Areas (BA) 9, 10, 45, 46]. In part, they extended to the medial surface of the prefrontal cortex $(\mathrm{BA} 32,24)$ or to the premotor area (BA 6, 8). In two cases, a glioblastoma infiltrated the anterior corpus callosum (No. 9, 18). In one case (No. 19), a large prefrontal stroke involved an additional small area of the anterior thalamus and the internal capsule. Patients with malign tumors were treated with steroids at the time of testing; their level of vigilance was normal.

Eight patients, six men and two women, had HD. Diagnosis was made on the basis of positive family history, clinical syndrome, brain imaging, and mutation analysis. Age ranged from 25 to 51 years. All patients were symptomatic with choreatic movements, but they were well able to perform the tasks that require movements, that is, Pursuit Rotor and the Tower of Hanoi. The functional staging score according to Shoulson and Fahn (1979) ranged from 1 to 4 (mean: 2.4). One patient was under risperidone therapy following an episode of psychosis.

Seven male, right-handed patients had a unilateral lesion to the head of the caudate nucleus (three right, four left hemisphere). Age ranged from 35 to 58 years. The lesions were ischemic in six cases and caused by hemorrhage in one. They were near complete in four cases and partial in three. Lesions involved the adjacent anterior limb of the internal capsula and the putamen in the four cases with left-side lesions. The delay between the onset of the lesions and inclusion in the study ranged from $2 \mathrm{wk}$ to $1 \mathrm{yr}$.

\section{Control Subjects}

Control group 1 consisted of 56 unpaid dermatological and other in-patients who were free of CNS disease or centrally active medication. Age ranged from 18 to $78 \mathrm{yr}$ (mean: 46.9). There were 32 men and 24 women. A subgroup of 40 subjects, aged 18 to $60 \mathrm{yr}$, had been included in a previous study (Schmidtke et al. 1996). Neuropsychological data are given in Table 3.

A second and independent group of 36 healthy normal subjects was recruited for the Pursuit Rotor task. There were 18 men and 18 women. Age ranged from 23 to 76 years (mean: 56.6). Neuropsychological tests were not applied in this group.

\section{General Design}

The 35 subjects with focal or degenerative fronto-striatal lesions were compared with control group 1 with regard to the cognitive tasks, and to control group 2 with regard to the Pursuit Rotor task. Subjects were trained in three trials on consecutive days. On each day, the tasks were administered one by one in fixed order. The measure of PL was the progress made from the baseline trial to the third trial, except for Tower of Hanoi and Pursuit Rotor (see below). PL was analyzed with regard to differences between groups and with regard to the role of cognitive abilities and age. PL was quantified in a manner that allows the application of parametric statistical methods, including calculation of Pearson's correlation coefficients, t-Tests, and analysis of variance.

\section{Procedural Learning Tasks}

Five cognitive and one visuo-motor task were applied in parallel as follows: Tower of Hanoi, Logical Reasoning, Mental Rotation, Mir-

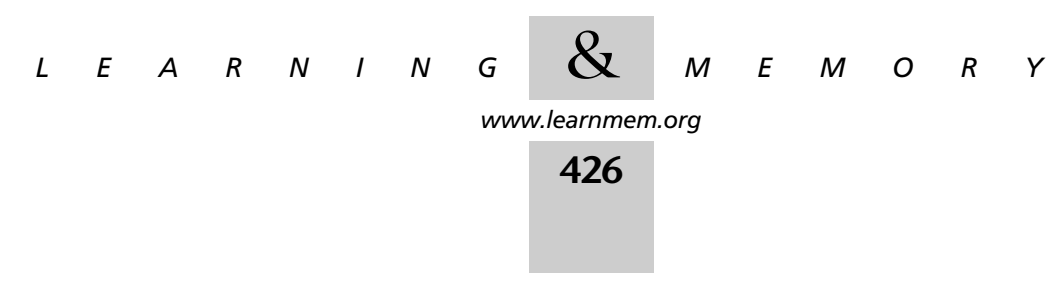


Table 4. Demographic and Lesion Data in the Group of Patients with Prefrontal Lesions

\begin{tabular}{|c|c|c|c|c|}
\hline $\begin{array}{l}\text { Patient no., } \\
\text { sex, age }\end{array}$ & Type of lesion & $\begin{array}{l}\text { Brodmann areas } \\
\text { affected by lesion }\end{array}$ & $\begin{array}{c}\text { Approximate } \\
\text { lesion size }(\mathrm{cm})\end{array}$ & $\begin{array}{l}\text { Time since onset } \\
\text { or operation }\end{array}$ \\
\hline $1, f, 67 y$ & Ischemic stroke & Left 8,9 & $4 \times 2 \times 2$ & $14 d$ \\
\hline $2, \mathrm{~m}, 61$ y & Circumscribed traumatic lesion & Right $8,9,32,45,46$ & $4 \times 2 \times 2.5$ & $\begin{array}{l}40 \mathrm{yr} \text {, epilepsy since } \\
\text { three months }\end{array}$ \\
\hline $3, m, 60 y$ & Meningeoma & Left $9,10,32,45,46$ & $4 \times 4 \times 4$ & Few days \\
\hline $4, m, 60 y$ & Hemorrhage & Left $9,10,45,46$ & $5 \times 3 \times 4$ & $5 \mathrm{~m} 5 \mathrm{yr}$ \\
\hline $5, f, 53$ y & Ischemic stroke & Left $8,9,10,24,32$ & $3 \times 4 \times 6$ & $20 \mathrm{yr}$ \\
\hline $6, m, 57 y$ & Ischemic stroke & Left $8,9,24$ & not available & 1 mon \\
\hline $7, f, 46 y$ & $\begin{array}{l}\text { Resection of Astrocytoma } I^{\circ} \text {, } \\
\text { progression }\end{array}$ & $\begin{array}{l}\text { Right } 8,9,10,44,45,46 \text {, } \\
24,32\end{array}$ & $4 \times 5 \times 6$ & $16 \mathrm{yr}$ \\
\hline $8, m, 46$ y & Glioblastoma & Left $9,10,45,46$ & $3 \times 4 \times 4$ & $6 w k$ \\
\hline $9, f, 59$ y & Glioblastoma & $\begin{array}{l}\text { Left } 8,9,10,24,32 \\
\text { anterior corpus callosum }\end{array}$ & $2 \times 3 \times 5$ & 1 mon \\
\hline $10, f, 26 y$ & Oligoastrocytoma IIIㅇ & Right 8,9 & $2 \times 2 \times 2$ & 6 mon \\
\hline $11, f, 63 y$ & Carcinoma metastasis & Right 44,45 & $2 \times 3 \times 3$ & $10 \mathrm{~d}$ \\
\hline $12, \mathrm{~m}, 32 \mathrm{y}$ & Astrocytoma ${ } I^{\circ}$ & Left 8,9 & $1.5 \times 2 \times 2.5$ & 16 mon \\
\hline $13, m, 36 y$ & Resection of meningeoma & Right $44,45,46$ & $4 \times 4 \times 5$ & 20 mon \\
\hline $14, f, 70 y$ & Resection of meningeoma & Right $6,8,9$ & $5 \times 3 \times 3.5$ & 18 mon \\
\hline $15, f, 50 y$ & Resection of meningeoma & Left $6,8,9,44,45,46$ & $6 \times 4 \times 4$ & 17 mon \\
\hline $16, f, 54 y$ & Resection of astrocytoma $\|^{\circ}$ & Right $6,8,9,10,24,32$ & $6 \times 4 \times 4$ & 13 mon \\
\hline $17, f, 49 y$ & Resection of meningeoma & Right $8,9,24,32,45,46$ & $5 \times 6 \times 7$ & 30 mon \\
\hline $18, f, 60 y$ & Glioblastoma & $\begin{array}{l}\text { Left } 8,9,10,24,32 \text {, anter. } \\
\text { corpus callosum }\end{array}$ & $4 \times 6 \times 7$ & $1 \mathrm{wk}$ \\
\hline $19, f, 21 y$ & Ischemic stroke & Right, 8, 9, 10, 24, 32 & $3 \times 4 \times 6$ & 2 mon \\
\hline $20, m, 62$ y & Resection of astrocytoma III & Left $9,10,24,32$ & $3 \times 3 \times 6$ & 10 mon \\
\hline
\end{tabular}

ror Reading, Sentence Arrangement, and Pursuit Rotor. Measure of performance was time-to-completion of all items in the cognitive tasks, and time-off-target in Pursuit Rotor. Different parallel versions of the tasks were used during each trial except for Tower of Hanoi and Pursuit Rotor.

Two reasoning and problem-solving tasks were administered, one verbal (Logical Reasoning) and one nonverbal (Tower of Hanoi). Two tasks require an attentional visuo-spatial procedure that is uniform, repetitive, and almost immediately evident (Mental Rotation and Mirror Reading). A fifth task of language processing requires neither of these processing types (Sentence Arrangement). A previous study with 20 amnesic patients showed no significant effects of declarative learning on the acquisition of these tasks (Schmidtke et al. 1996). However, it remains possible that declarative learning of certain strategies can contribute to the learning of the Tower of Hanoi task.

As a measure of PL, neither the performance on the third trial nor the difference between baseline and third trial appeared suitable, because these measures are biased by the individual level of baseline performance. In the tasks applied here, controls' baseline and third trial scores exhibit a close linear relationship (see Fig. $1 \mathrm{~A}, \mathrm{~B})$. This indicates a tight proportionality between individual preand post-training performances. The quotient of third day performance divided by first day performance was therefore applied as a the measure of PL. It is referred to as procedural learning score (see Schmidtke et al. 1996). The principle advantage of this measure of PL is its independence of the individual level of performance at baseline. It is applicable up to the point when subjects begin to approximate a ceiling level of performance.

As exemplified by Figure 1, A and B, the regression lines cross the y-axis shortly above the origin. This leads to a small bias toward numerically higher quotients (i.e., poorer learning) in subjects with numerically low (i.e., good) baseline scores. To remove this bias, a transformation is carried out. A small constant is subtracted from all third trial scores, so that the controls' regression lines cross the origin. The resultant learning scores range from $\sim 0.3$ to 1 , that is, $70 \%$ improvement to no improvement at all. In single cases in which post-training performance was actually lower than baseline performances, the learning score was set to 1 , that is, no learning. Single patients whose baseline score was below three standard deviations of the controls' means were excluded taskwise from the analysis, because learning may occur in a qualitatively different manner in very low-performing subjects (two cases each in Tower of Hanoi, Logical Reasoning, and Mirror Reading, five cases in Mental Rotation, one case in Sentence Arrangement).

\section{Tower of Hanoi}

This task was performed with four wooden disks having to be moved from the left pole to the right pole without placing a larger disk above a smaller one or moving more than one disk at a time. The minimum number of moves is 15 . The task was performed twice per trial, once at the beginning and once at the end of the session (six trials total). Rule violations were corrected immediately. This task involves planning and problem solving, specifically at the early stage of learning. The principle measure of performance was the time required to solve the problem. The number of moves $>15$ required to complete the task was used as an additional measure. As most subjects learn this task fast, the quotient of second versus first trial performances of the first day was used as the principle measure of PL. Two female patients of the prefrontal group with left-side glioblastoma that extended to the anterior corpus callosum could not solve the task.

\section{Logical Reasoning}

Each trial of this task involved 12 problems, which consisted of 2 written statements printed on cards describing the relationship

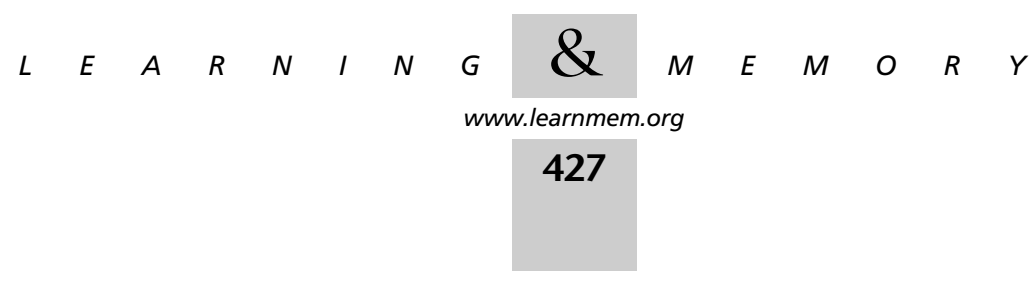


between 3 subjects or objects $\mathrm{A}, \mathrm{B}$, and $\mathrm{C}$, and a question whose answer could be deduced from the statements. The relationships were concrete in nature, for example, (1) A is heavier than $\mathrm{B}$, but lighter than $\mathrm{C}$. Who is the lightest? (2) A is much older than B. B is a little younger than $\mathrm{C}$. Who is the youngest? (3) A has more money than $\mathrm{B}$. C has less money than $\mathrm{A}$. Who has the most money?

Different relationships were used for each item. There were six different types of logical constructions. Each was applied twice per trial. Subjects were encouraged to convert the written statements into representational mental images of size. The use of paper and pencil was allowed. When an incorrect solution was offered, the corresponding card was put at the bottom of the stack, until all problems were solved correctly. The three parallel versions of this task involved the same objects, relationships and logical constructions, but their combinations varied. This task involves reasoning and problem solving, as well as visuo-spatial processing, because subjects have to convert the written statements into representational mental images of size relationships.

\section{Sentence Arrangement}

This task involved 20 problems per trial. Each contained five or six nonsensically ordered words that had to be arranged to form a meaningful and grammatically correct sentence in indirect speech. The sentence then had to be read aloud. Only one correct arrangement was possible. All sentences began with a personal pronoun, which was printed below as a prompt, for example,

anderes meine Redner glaubten etwas der Wir............ (else meant speaker thought something the We.........)

Three patients refused to perform the task; one whose native language was not German and two for unspecified reasons.

\section{Mental Rotation}

Each trial involved five sheets with a different asymmetric symbol printed at the top of each (reference symbol). Below this, 16 alternative views of this same symbol were arranged in a $4 \times 4$ fashion. These varying views were achieved by rotating the reference symbol to different degrees. Eight of the sixteen views were also inverted. The instructions were printed at the bottom of each sheet: "Mark those symbols which can be derived from the reference symbol by rotation only." For every error made, one-sixteenth of the time needed for this sheet was added. Whereas the same symbols were used in the three parallel versions of this task, the $4 \times 4$ arrangements of symbols were rotated and oriented in a different manner from trial to trial. The following symbols were used: a pictogram symbolizing a wheel chair driver, the silhouette of a bird, the hammer and sickle emblem, a typographic version of the Figure 1 , and a flag.

\section{Mirror Reading}

Although sometimes referred to as perceptual, this task bears an obvious cognitive component. Namely, during untrained performance, it involves attention and effort for the visuo-spatial transformation of symbols, storage of decoded letters in working memory, and their composition to words. In the present paradigm, each trial was composed of eight lists of ten six-to-eight-letter words. Each word was presented only once. The words were printed mirror reverse with a horizontal mirror axis, that is, upsidedown (but not backward). This type of visual transformation is more difficult than lateral mirror images and rotations of words or individual letters. Subjects had to read the words aloud. Words that were not read correctly had to be attempted again at the end of the corresponding list. Four patients of the prefrontal group experienced this task as too strenuous and gave up before the end of the first trial.

\section{Pursuit Rotor}

The apparatus (Schuhfried, Mödlingen/Austria) consists of a box covered by a glass plate that is opaque except for a heptagonal trail along which the light stimulus moves clockwise at variable speed (target). The diameter of the heptagon is $\sim 20 \mathrm{~cm}$. Subjects have to follow a moving stimulus with a light-sensitive pen, using their dominant hand. The time on- and off-target was registered, and time off-target was the measure of performance. Each trial lasted $60 \mathrm{sec}$, with a break after $30 \mathrm{sec}$. Two trials were performed per day (six trials total). To avoid floor and ceiling effects, the speed of the moving light stimulus, that is, number of rounds per minute, was adjusted individually to 10,15 , or 20 rounds per minute, so that pretraining time off-target was between 10 and $20 \mathrm{sec}$ during a 30 -sec period. This speed was kept unchanged during all trials. The mean speed was similar in all groups (Table 1, sixth line). Compared with other tasks, there was a high variability of performances from trial to trial. To reduce variability, the means of the times-offtarget were calculated for the first three trials and for the last three trials. As a measure of learning (PL score), the quotient of these two values was taken, that is, the mean of the last three trials divided by the mean of the first three trials.

\section{Visuo-Spatial Ability, Frontal/Executive Capacity and Other Neuropsychological Measures}

Cognitive abilities that were assumed to play a role in the processing and/or improvement of the PL tasks were evaluated by standard neuropsychological tests. As a measure of visuo-spatial ability, the sum of the scaled scores of the Block Design and the Object Assembly subtests of the revised version of the Wechsler Adult Intelligence Scale was used (WAIS-R; Tewes 1991). As a measure of frontal/executive capacity, a six-category word fluency test was used (animals, fruit, furniture, girls' names, birds, metals). Time per category was $1 \mathrm{~min}$; measure of performance was the sum of correct items for all categories. Additional descriptive measures that were applied in patients only were object naming (Aachen Aphasia Test, maximum score 60, Huber et al. 1983) and, as an estimate of intelligence, the sum of the scaled scores of three WAIS-R subtests (Arithmetic, Similarities, and Picture Completion).

\section{ACKNOWLEDGMENTS}

The cooperation of Professor B. Landwehrmeyer in the recruitment of patients with Huntington's disease is gratefully acknowledged.

The publication costs of this article were defrayed in part by payment of page charges. This article must therefore be hereby marked "advertisement" in accordance with 18 USC section 1734 solely to indicate this fact.

\section{REFERENCES}

Alexander, G.E. and Crutcher, M.D. 1990. Functional architecture of basal ganglia circuits: Neural substrates of parallel processing. Trends Neurosci. 13: 266-270.

Alexander, G.E., DeLong, M.R., and Strick, P.L. 1986. Parallel organization of functionally segregated circuits linking basal ganglia and cortex. Annu. Rev. Neurosci. 9: 357-382.

Alexander, G.E., Crutcher, M.D., and DeLong, M.R. 1990. Basal ganglia-thalamocortical circuits: Parallel substrates for motor, oculomotor, 'prefrontal' and 'limbic' function. Progr. Brain Res. 85: 119-146.




Anderson, J.R. 1982. Acquisition of cognitive skill. Psychol. Rev. 89: 369-406.

Baker, S.C., Rogers, R.D., Owern, A.M., Frith, C.C., Dolan, R.J., Frackowiak, R.S., and Robbins, T.W. 1996. Neuronal systems engaged by planning: A PET study of the Tower of London task. Neuropsychologia 34: 515-526.

Beatty, W.W., Salmon, D.P., Bernstein, N., Martone, M., Lyon, L., and Butters, N. 1987. Procedural learning in a patient with amnesia due to hypoxia. Brain Cogn. 6: 386-402.

Beldarrain, M.R., Grafman, J., Pascual-Leone, A., and Carcia-Monco, J.C. 1999. Procedural learning is impaired in patients with prefrontal lesions. Neurology 52: 1853-1860.

Brooks, D.N. and Baddeley, A. 1976. What can amnesic patients learn? Neuropsychologia 14: 111-122.

Butters, N., Wolfe, J., Martone, M., Granholm, E., and Cermak, L.S. 1985 Memory disorders associated with Huntington's disease: Verbal recall, verbal recognition and procedural memory. Neuropsychologia 23: 729-743.

Cummings, J.F. 1993. Frontal-subcortical circuits and human behavior. Arch. Neurol. 50: 873-880.

Dagher A., Owen, A.M., Boecker, H., and Brooks, D.J. 1999. Mapping the network for planning: A correlational PET activation study with the Tower of London task. Brain 122: 1973-1987.

Daum, I., Schugens, M.M., Spieker, S., Poser, U., Schönle, P.W., and Birbaumer, N. 1995. Memory and skill acquisition in Parkinson's disease and frontal lobe dysfunction. Cortex 31: 413-432.

De la Monte, S.M., Vonsattel, J.P., and Richardson E.P. 1988. Morphometric demonstration of atrophic changes in the cerebral cortex, white matter and neostriatum in Huntington's Disease. $J$. Neuropatbol. Exp. Neurol. 47: 516-525.

DeLong, M.R., Georgopoulos, A.P., and Crutcher, M.D. 1983. Cortico-basal ganglia relations and coding of motor performance. Exp. Brain Res 49: 30-40.

Gabrieli, J.D., Stebbins, G.T., Singh, J., Willingham, D.B., and Goetz, C.G. 1987. Intact mirror-tracing and impaired rotary-pursuit skill learning in patients with Huntington's disease: Evidence for dissociable memory systems in skill learning. Neuropsychology 11: 272-281.

Grafton, S.T., Mazziotta, J.C., Presty, S., Friston, K.J., Frackowiak, R.S., and Phelps, M.E. 1992. Functional anatomy of human procedural learning determined with regional cerebral blood flow and PET. J. Neurosci. 9: 582-587.

Harrington, D.L., Haaland, K.Y., Yeo, R.A., and Marder, E. 1990 Procedural memory in Parkinson's disease: Impaired motor but not visuoperceptual learning. J. Clin. Exp. Neuropsychol. 12: 323-339.

Heindel, W.C., Salmon, D.P., Shults, C.W., Walicke, P.A., and Butters, N. 1989. Neuropsychological evidence for multiple implicit memory systems: A comparison of Alzheimer's, Huntington's and Parkinsons disease patients. J. Neurosci. 9: 582-587.

Holthoff-Detto, V.A., Kessler, J., Herholz, K., Bonner, H., Pietrzyk, U., Wurker, M., Ghaemi, M., Weinhard, K., Watgner, R., and Heiss, W.D. 1997. Functional effects of striatal dysfunction in Parkinson Disease. Arch. Neurol. 54: 145-150.

Huber, W., Poeck, K., Weniger, D., and Willmes, K. 1983. Aachener Aphasie Test (AAT). (ed. C.J. Hogrefe). Göttingen, Toronto, Zürich.

Kassubek, J., Schmidtke, K., Kimmig, H., Lücking, C.H., and Greenlee, M. 2001. Changes in cortical activation during mirror reading before and after training: An fMRI study of procedural learning. Brain Res. Cogn. Brain Res. 10: 207-217.
Koenig, O., Thomas-Antérion, C., and Laurent, B. 1999. Procedural learning in Parkinson's disease: Intact and impaired cognitive components. Neuropsychologia 37: 1103-1109.

Martone, M., Butters, N., Payne, M., Becker, J.T., and Sax, S.S. 1984. Dissociation between skill learning and verbal recognition in amnesia and dementia. Arch. Neurol. 41: 965-970.

Peigneux, P., Maquet, P., Meulemans, T., Destrebecqz, A., Laureys, S., Dequeldre, C., Delfiore, G., Aerts, J., Luxen, A., Franck, G., et al. 2000. Striatum forever, despite sequence learning variability: A random effect analysis of PET data. Hum. Brain Mapp. 10: 179-194.

Perani, D., Bressi, S., Cappa, S.F., Vallar, G., Alberoni, M., Grassi, F., Caltagirone, C., Cipolotti, L., Franceschi, M., Lenzi, G.L., et al. 1993. Evidence of multiple memory systems in the human brain. An [18F] FDG PET metabolic study. Brain 116: 903-919.

Poldrack ,R.A., Desmond, J.E., Glover, G.H., and Gabrieli, J.D.E. 1998. The neural basis of visual skill learning. An fMRI study of mirror-reading. Cereb. Cortex 8: 1-10.

Poldrack, R.A., Prabhakaran, V., Seger, C.A., and Gabrieli, J.D. 1999. Striatal activation during acquisition of a cognitive skill. Neuropsychology 13: 564-574.

Reber, P.J. and Squire, L.R. 1999. Intact learning of artificial grammars and intact category learning by patients with Parkinson's Disease. Behav. Neurosci. 113: 235-242.

Roncacci, S., Troisi, E., Carlesimo, G.A., Nocentini, U., and Caltagirone, C. 1999. Implicit memory in Parkinsonian patients: Evidence for deficient skill learning. Eur. Neurol. 36: 154-159.

Saint-Cyr, J.A. and Taylor, A.E. 1992. The mobilization of procedural learning: The "Key Signature" of the basal ganglia. In Neuropsychology of memory, 2nd ed, (eds. L.R. Squire and N. Butters), pp. 188-202. Guilford Press, New York.

Saint-Cyr, J.A., Taylor, A.E., Trépanier, L.L., and Lang A.E.. 1992. The caudate nucleus: Head ganglion of the habit system. In Neuropsychological disorders associated with subcortical lesions, (eds. G. Vallar, S.F. Cappa, and C.-W. Wallesch). pp. 205-223. Oxford University Press, Oxford, New York, Tokyo.

Saint-Cyr, J.A., Taylor, A.E., and Nicholson, K. 1995. Behavior and the basal ganglia. In Behavioral neurology of movement disorders, (eds. W.J. Weiner and A.E. Lang) pp. 1-28. Advances in Neurology, Vol. 65, Raven Press, New York.

Saint-Cyr, J.A., Taylor, A.E., and Lang, A.E. 1998. Procedural learning and neostriatal dysfunction in man. Brain 111: 941-959.

Schmidtke, K., Handschu, R., and Vollmer, H. 1996. Cognitive procedural learning in amnesia. Brain Cogn. 32: 441-467.

Shoulson, I. and Fahn, S. 1979. Huntington disease: Clinical care and evaluation. Neurology 29: 1-3.

Sotrel, A., Paskevich, D.A., Kiely, D.K., Bird, E.D., Williams, R.S., and Myers, R.H. 1991. Morphometric analysis of the prefrontal cortex in Huntington's Disease. Neurology 41: 1117-1123.

Tewes, U. (Ed.). 1991. Hamburg-Wechsler Intelligenztest für Erwachsene. Revision. Bern, Stuttgart, Toronto: Hans Huber.

van Mier, H., Tempel, L.W., Perlmutter, J.S., Raichle, M.E., and Petersen, E.S. 1998. Changes in brain activity during motor learning measured with PET: Effects of hand of performance and practice. $J$. Neurophysiol. 80: 2177-2199.

Received January 31, 2002; accepted in revised form August 22, 2002.

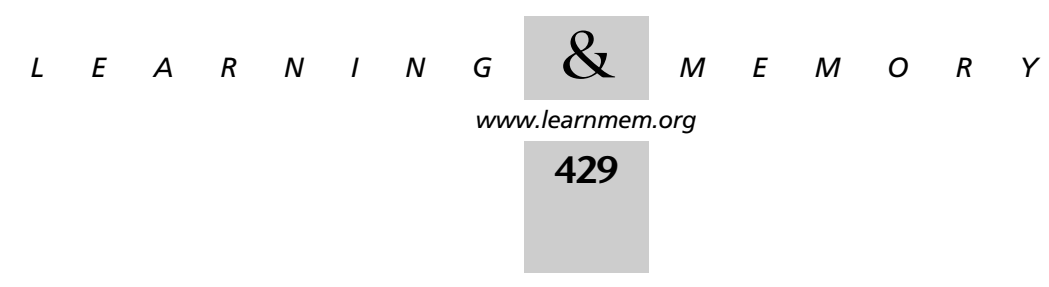




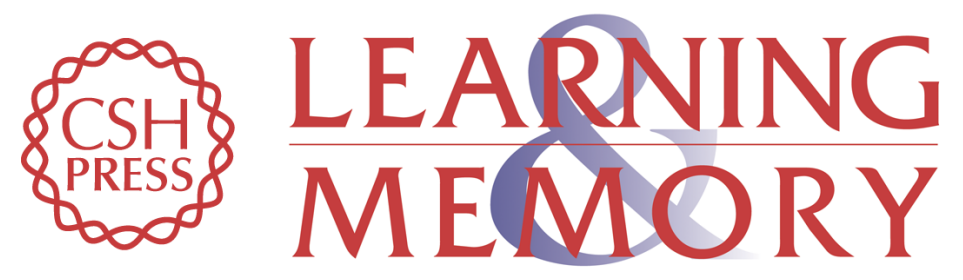

\section{Cognitive Procedural Learning in Patients With Fronto-Striatal Lesions}

Klaus Schmidtke, Hendrik Manner, Robert Kaufmann, et al.

Learn. Mem. 2002, 9:

Access the most recent version at doi:10.1101//m.47202

\section{References This article cites 31 articles, 4 of which can be accessed free at: http://learnmem.cshlp.org/content/9/6/419.full.html\#ref-list-1 \\ License}

Email Alerting

Receive free email alerts when new articles cite this article - sign up in the box at the top Service right corner of the article or click here. 